\title{
Evolution Du Couvert Végétal Naturel Au Niveau Des Massifs Forestiers De Mallalyine Et Taghramt (Rif Occidental Du Maroc)
}

\author{
Mhammad Houssni \\ Laboratoire Botanique Appliquée, Equipe Agrobiodiversité. Faculté des \\ Sciences, Université Abdelmalek Essaâdi. Tétouan, Maroc.
}

Hassan Ennouni

Direction des Eaux et Forêts. 33, Av. Mohamed V, Tétouan, Maroc

\section{Abdessalam Ouallali}

Laboratoire de Géologie de l'Environnement et Ressources Naturelles, Equipe Cartographie et Technologie Numérique. Faculté des Sciences, Université Abdelmalek Essaâdi. Tétouan, Maroc.

\section{Jalal Kassout Mohamed El Mahroussi Abdelouahab Sahli \\ Hasnae Bensbih Mohamed Kadiri Mohammed Ater}

Laboratoire Botanique Appliquée, Equipe Agrobiodiversité. Faculté des Sciences, Université Abdelmalek Essaâdi. Tétouan, Maroc.

\begin{abstract}
This work presents the results of a cartographic study of the spatiotemporal evolution of vegetation cover in the forest areas of Mallalyine and Taghramt in the western Rif. It is based on a field survey and the photointerpretation of aerial photographs taken in 1986 and Google Earth images from 2013 (a period of twenty-seven years). Our study reports on the current state of degradation of the vegetation cover in the forests of Mallalyine and Taghramt. We have produced two land use maps showing how high and low formations as well as scrub have regressed by 62,70 and 49 percent, respectively, between 1986 and 2013, while non-forested land and reforestation increased respectively by 26.3 and 1.53 percent relative to the area covered in 1986. The main factors contributing to the decline of vegetation cover are: deforestation (35 offenses registered every year with a degraded area of about 15 ha / year), clearing ( 27 offenses per year with an
\end{abstract}


affected area of about 20 ha / year), overgrazing (10 offenses of illegal grazing per year with a degraded area of about 12 ha / year), forest fires (4 to 5 fires per year with a degraded area of about 50 ha / year). In addition to these factors, we find increasing water scarcity.

Keywords: Vegetation, degradation, forests, photo-interpretation, GIS, western Rif, Morocco.

\section{Résumé}

Le présent travail expose les résultats d'une étude cartographique analysant l'évolution spatio-temporelle du couvert végétal au niveau des massifs forestiers de Mallalyine et Taghramt localisés dans le Rif occidental (Maroc). Il est basé sur une prospection du terrain et l'interprétation de photographies aériennes de la mission de 1986 et des images satellites de type Landsat 8 acquises sur Google Earth 2013. L'étude fait le point sur l'état actuel de la dégradation du couvert végétal dans les massifs forestiers de Mallalyine et Taghramt sur une période de 27 ans. Elle a produit deux cartes d'occupation des sols en utilisant les techniques de spatialisation telles que les SIG et la télédétection. Cette étude montre que les formations hautes, les formations basses et le matorral ont régressé respectivement de $62 \%, 70 \%$ et 49\% entre les années 1986 et 2013. Les terrains non boisés et les reboisements se sont accrus respectivement de $26,3 \%$ et $1,53 \%$ par rapport à la superficie qu'ils occupaient en 1986. Les principaux facteurs contribuant à la régression du couvert végétal sont: les prélèvements délictueux du bois (35 délits de coupe par an avec une superficie dégradée de l'ordre de 15 ha/an), le défrichement (27 délits par an avec une superficie dégradée de l'ordre de 20 ha/an), le surpâturage (10 délits de pâturage illicite par an avec une superficie dégradée de l'ordre de $12 \mathrm{ha} / \mathrm{an}$ ), les incendies de forêts (de 4 à 5 incendies par an avec une superficie dégradée de l'ordre de 50 ha/an). En plus de ces facteurs s'ajoute l'érosion hydrique. Les objectifs de cette étude consistent à tracer l'évolution du couvert végétal au niveau des massifs forestiers de Mallalyine et Taghramt (Rif occidental) ; à la caractérisation des différentes strates qui apparaissent dans la zone d'étude; à l'interprétation de photographies aériennes (mission Mars 1986), des images Google Earth (Mars 2013) et des observations sur le terrain et à l'identification des facteurs à l'origine de la réduction du couvert végétal à l'échelle des deux massifs.

Mots clés: Couvert végétal, dégradation, massif forestier, photointerprétation, Rif occidental, Maroc, S.I.G. 


\section{Introduction}

Au niveau de la méditerranée les forêts ont joué un rôle important dans le développement des populations (Tillier, 2011). Les forêts méditerranéennes ont été exploitées pour leurs différents usages et appréciées pour les multiples biens et services qu'elles procurent à ces populations (Marien \& Billand, 2009). Cependant, la surexploitation a entraîné aujourd'hui la dégradation des forêts dans de nombreux espaces méditerranéens (FAO, 2013). En 2010, la surface forestière des pays méditerranéens était estimée à 85 millions d'hectares, représentant environ $2 \%$ de la surface forestière mondiale (4 033 millions d'hectares) (FAO, 2013). Au Maroc les formations forestières naturelles, y compris les nappes alfatières, couvrent près de 9 millions d'hectares, et se trouvent essentiellement dans des bioclimats subhumides, arides et semi-arides (M'hirit \& Benchekroun, 2006). Les boisements artificiels, pour leur part, s'étendent sur une superficie de 530.000 hectares, alors que les terres de pâturages couvrent près de 53 millions d'hectares, situés pour la plupart dans les zones à bioclimat aride, présaharien et saharien (Benabid, 2000; Narjisse et al, 2001). Cet ensemble représente une richesse floristique considérable, comprenant plus de 4500 espèces et sous-espèces dont 600 endémiques (Fennane \& Ibn Tattou, 1998 ; Fennane \& Ibn Tattou 2005 ; Fennane, 2003). La forêt marocaine produit particulièrement et annuellement 1,6 millions $\mathrm{m}^{3}$ de bois d'œuvre, 4500 à 5000 tonnes de liège et 1,5 milliards d'unités fourragères (AEFCS, 1995). Elle permet aussi aux populations riveraines de s'approvisionner en bois de feu et de services (AEFCS, 1995). Dans les régions rifaines, les parcours, y compris les formations forestières, occupent une superficie évaluée à quelques 900.000 hectares soit $1,5 \%$ de la superficie totale des parcours nationaux (Berkat, 1995). Ces écosystèmes terrestres ont une végétation le plus souvent, sous forme de matorral, caractérisée par une richesse floristique et dominée par de nombreuses espèces endémiques (Benabid, 1982). En effet, la diversité climatique et lithologique qui caractérise les régions du Rif, font que cette portion de l'Afrique du Nord offre les meilleures possibilités pour le développement des potentialités forestières et pastorales (Qarro, 1996a ; Kouraimi, 1997). Toutefois, ces espaces risqueraient de disparaitre si la poussée démographique persistait. Le territoire rifain, qui représente 5,5\% du territoire national, est occupé par $15 \%$ de la population nationale avec une densité qui peut aller parfois jusqu'à 230 ha/ $\mathrm{km}^{2}$ (Kouraimi, 1997). Cette surpopulation des régions rifaines aurait pour conséquence une surexploitation du milieu (incendies, mise en culture, défrichement, surpâturage...) dont la résultante est la régression du couvert végétal (Benabid, 2002; Benabid, 1984). Chaque année environ 463 ha des matorrals rifains sont dégradés par les incendies et 398 ha par les défrichements (Boukil in El Haddad, 1993). Dans le présent travail, nous faisons usage d'un système d'information 
géographique (SIG) dont l'objectif général est de tracer l'évolution du couvert végétal au niveau des massifs forestiers de Mallalyine et Taghramt (Rif occidental). Les objectifs spécifiques du travail consistent à la caractérisation des différentes strates qui apparaissent dans la zone d'étude ; à l'interprétation de photographies aériennes (mission Mars 1986), des images Google Earth (Mars 2013) et des observations sur le terrain et à l'identification des facteurs responsable de la réduction du couvert végétal à l'échelle des deux massifs.

\section{Présentation de la zone d'étude}

Les massifs forestiers de Mallalyine (Latitude: -5.3306014 et Longitude : 35.6256868) et Taghramt (Latitude : -5.4699173 et Longitude 35.7883462) se situent au cœur de la péninsule Tingitane (El Gharbaoui, 1981). La zone d'étude s'attache à la forêt d'El Haouz (El Gharbaoui, 1986) qui est située au Nord de la ville de Tétouan et domine le littoral entre cette ville et Fnideq. La zone d'étude couvre une superficie de 11425ha. Selon le découpage administratif établi au début de 2009 la zone d'étude s'étend sur trois provinces et préfectures que sont: Tétouan, Fahs-Anjra et Mdiq-Fnideq (Figure 1). La zone d'étude recoupe les limites administratives de cinq communes rurales dont trois couvrent plus de $90 \%$ de la superficie totale: Alléyinne, Mallaliyine et Taghramt.

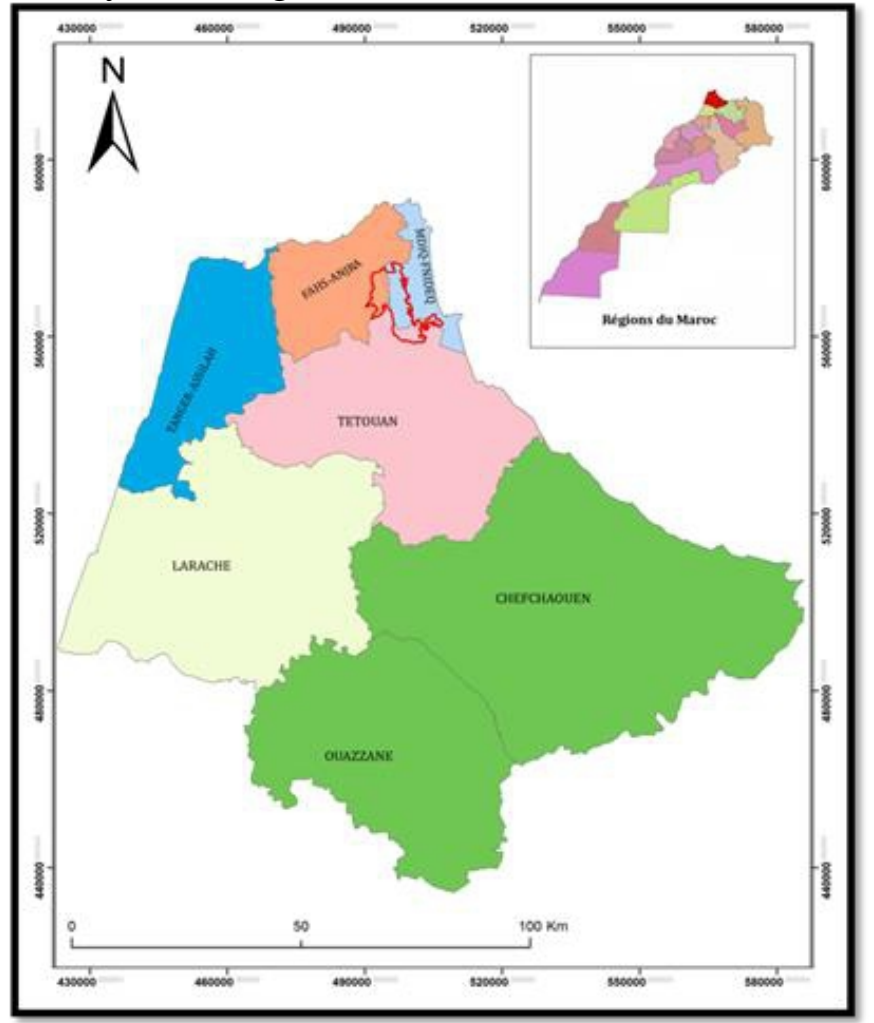

Figure 1 : Découpage administratif régional de la zone d'étude. 


\section{Méthodologie du travail}

En principe, deux technologies sont inéluctables pour assurer ce travail : la photo-interprétation caractérisée par sa vision synoptique et les systèmes d'informations géographiques reconnus notamment pour leur performance en matière de rapidité d'analyse spatiale. Le couvert végétal des massifs forestiers de Mallalyine et Taghramt était sujet à une régression très intense au cours d'une période (1986-2013). Pour savoir si ce changement est lié à une imbrication des facteurs anthropiques, on a adopté une méthodologie reposant sur l'évaluation du développement des terrains de cultures au détriment des formations végétales naturelles entre 1986 et 2013. Pour réaliser ceci, la superposition des 2 cartes du couvert végétal réalisées pour les deux dates était nécessaire pour extraire et localiser les extensions des cultures. Les strates dégagées après la photo interprétation et les contrôles sur le terrain sont: formation haute (hauteur supérieure à $3 \mathrm{~m}$ ) ; formation basse (hauteur inférieure à $3 \mathrm{~m}$ ) ; matorrals ; reboisement (âge plus de 3ans et de plus de 250 pieds/ha) et terrain non boisé (cette classe comporte à la fois les terrains de culture, les agglomérations et les affleurements rocheux).

Les documents de base utilisés dans cette étude sont : Carte topographique de Mellousa 1/50000ème (ANCFCC, 2007);Carte topographique de Tétouan 1/50000éme (DCFTT, 1970) et 1/25000éme (ANCFCC, 2007);Carte topographique de Sebta 1/50000éme (DCFTT, 1970);Les photographies aériennes de mission 1986 1/20000éme. La délimitation de la zone d'étude a été faite grâce à l'outil SIG-QGis 2.10. Ce dernier permet le traitement des images satellitaires par une classification supervisée basé sur l'approche des plus proches voisins. Il s'agit d'un algorithme de stockage des données d'apprentissage qui permet de comparer une donnée de classe inconnue à toutes les données stockées (Figure 2).

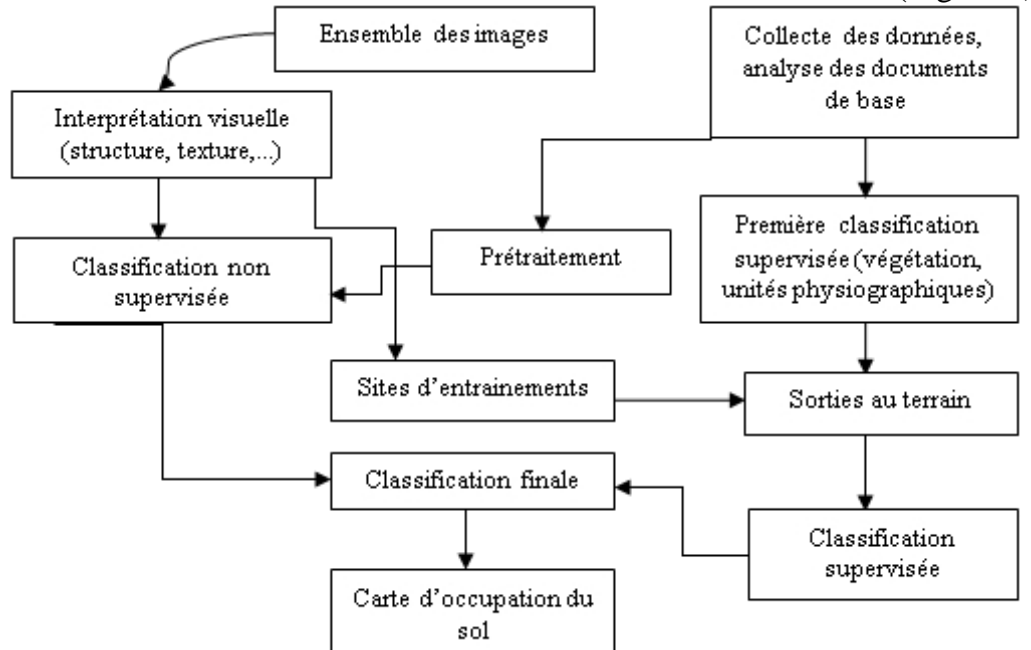

Figure 2 : Schéma montrant les différentes étapes de la méthodologie adoptée pour le travail. 
Le contrôle et la vérification sur le terrain consistent à vérifier la stratification préétablie. Elle permet également de rectifier, corriger et apporter les éléments d'informations jugés utiles afin d'avoir une stratification définitive. Le tableau 1 présente le nombre de sites d'entrainement visités et leurs coordonnées géographiques.

Tableau 1: Points de contrôle sur le terrain de la zone d'étude

\begin{tabular}{|c|c|c|c|c|c|}
\hline Site & Latitude & Longitude & Site & Latitude & Longitude \\
\hline 1 & $35.764313^{\circ}$ & $-5.471287^{\circ}$ & 11 & $35.692910^{\circ}$ & $-5.422439^{\circ}$ \\
\hline 2 & $35.761777^{\circ}$ & $-5.465123^{\circ}$ & 12 & $35.691714^{\circ}$ & $-5.410528^{\circ}$ \\
\hline 3 & $35.759788^{\circ}$ & $-5.461135^{\circ}$ & 13 & $35.673705^{\circ}$ & $-5.407231^{\circ}$ \\
\hline 4 & $35.767207^{\circ}$ & $-5.443750^{\circ}$ & 14 & $35.666882^{\circ}$ & $-5.412605^{\circ}$ \\
\hline 5 & $35.772741^{\circ}$ & $-5.427332^{\circ}$ & 15 & $35.663950^{\circ}$ & $-5.397317^{\circ}$ \\
\hline 6 & $35.721527^{\circ}$ & $-5.489523^{\circ}$ & 16 & $35.661508^{\circ}$ & $-5.391610^{\circ}$ \\
\hline 7 & $35.725688^{\circ}$ & $-5.460822^{\circ}$ & 17 & $35.671364^{\circ}$ & $-5.366269^{\circ}$ \\
\hline 8 & $35.731633^{\circ}$ & $-5.435914^{\circ}$ & 18 & $35.656376^{\circ}$ & $-5.378083^{\circ}$ \\
\hline 9 & $35.727361^{\circ}$ & $-5.409062^{\circ}$ & 19 & $35.643340^{\circ}$ & $-5.394771^{\circ}$ \\
\hline 10 & $35.700252^{\circ}$ & $-5.408741^{\circ}$ & 20 & $35.648657^{\circ}$ & $-5.426796^{\circ}$ \\
\hline
\end{tabular}

\section{Résultats et discussions}

La méthodologie adoptée a permis d'élaborer deux cartes d'occupation des sols des deux massifs forestiers de Mallalyine et Taghramt, celle de 1986 et 2013 .

\section{Description des unités cartographiques Formation haute:}

Ce sont des peuplements dont la taille est supérieure à $3 \mathrm{~m}$, constitués par Quercus suber (chêne liège) et l'Olea europea (l'oléastre), qui sont des peuplements localisés sur des substrats marneux et Quercus coccifera (chêne kérmès) et Tetraclinis articulata (Thuya) sur des substrats calcaires. Ces formations hautes sont surtout localisées autour de certains marabouts ou des pentes inaccessibles pour l'homme, elles ne couvrent actuellement que 292 ha. Ces formations sont localisées au centre et au nord de notre zone d'étude, en particulier suivant deux chainons montagneux parallèles, le premier vers l'est et l'autre vers l'ouest. Elles représentent les témoins d'une végétation originale qui dominait cette région. Ce type de formation est constitué d'un cortège floristique très diversifié à base de: Arbutus unedo, Erica arborea, Pistacia lentiscus, Myrtus communis, Cistus monspeliensis, Cistus populifolius, Cistus albidus, Cistus salviifolius, Chamaerops humilis, Phyllirea angustifolia.

\section{Formation basse:}

Ce sont des formations dont la taille est inférieure à $3 \mathrm{~m}$, s'étendant uniquement sur une superficie de 342 ha. Elles sont à la base de Quercus 
coccifera, Quercus suber, Tetraclinis articulata, Erica arborea, Pistacia lentiscus, Arbutus unedo.

Ces formations sont éparpillées sur tout le territoire de la zone d'étude sur des strates plus au moins importantes et entourées par de larges espaces de matorral.

\section{Matorral:}

Le matorral occupe une superficie de l'ordre de 17,54\% soit 2004 ha par rapport à la superficie totale de la zone d'étude, il est localisé partout dans la zone d'étude. D’après Benabid (1982), cette formation dérive de la dégradation de la forêt du fait des incendies, des coupes de bois, du surpâturage, etc. Ce matorral est constitué par des groupements très variés, qui bien que très influencés par l'action humaine témoignent de la végétation originelle du milieu : lentisque et calycotome caractérisent les terrains marneux; le doum vient sur des matériaux argileux et argilo-calcaires ; les Cistacées et Ericacées sont les plus répandues. Les Cistaies sont à ciste de montpellier, ciste ladanifère, ciste crépu en terrains superficiels, ciste à feuille de peuplier. Quant aux Ericacées, E. arborea et arbousier indiquent les meilleurs sols, les autres, les xérophiles (E. umbellata, E. scoparia, callune) croissent sur les terrains les plus rocheux, dont la première est la plus caractéristique.

\section{Reboisement:}

Les reboisements réalisés par le service des Eaux et Forêts visent surtout la protection du sol, la production du bois, la reconstitution des écosystèmes naturels à savoir le chêne liège et le thuya et aussi pour essayer de compenser le recul du domaine forestier dans cette zone. Ils occupent 940,5 ha environ $9 \%$ de la superficie totale de la zone d'étude. Les essences utilisées pour le reboisement sont les suivantes :

- Les pinèdes : ces peuplements denses purs ou en mélanges avec le chêne liège sont composés de : Pinus pinaster var. moghrebiana et iberica, Pinus radiata, Pinus pinea, Pinus halepensis et Pinus canariensis. Il se situe dans la partie Nord et Sud-est de la zone. Il s'agit des périmètres de Dar Miman, Boujmil, Touilaa, Ghniouat, Kalaa Koudiat Taïfor et Oued Ellile. Ces périmètres ont été reboisés entre 1942-1998.

- Acacia cyanophylla : dont le reboisement, réalisé en 2009, a été effectué dans le périmètre de Ghniouat. Le choix de cette essence a été guidé par sa croissance rapide et par le désir d'associer une utilisation pastorale au reboisement.

- Eucalyptus camaldulensis : a été planté essentiellement dans le but de stabiliser les sols. Il se situe dans la partie Sud-est de la zone dans les 
périmètres de Koudiat Taïfor en 1942, Ghniouat en 1998 et 2008, Oued Ellile en 1972 et dans le périmètre de Kalaa en 2008.

Il y a lieu de préciser que les critères de l'inventaire forestier national précisent qu'un reboisement acquis et pris en considération dans les bilans de gestion doit avoir au moins 3 ans et une densité de 250 pieds/ha; le critère de taille n'est pris en considération que pour les formations naturelles.

\section{Terrain non boisé:}

Cette strate occupe une superficie importante plus de 50\% de la zone d'étude. On peut distinguer dans cette strate les terrains de culture, les agglomérations et les affleurements rocheux. Les agriculteurs pratiquent surtout une céréaliculture à base de culture annuelle de blé, orge, en rotation avec le maiis.

\section{Résultat de l'étude multidates du couvert végétal (1986-2013)}

- Planimétrie des deux cartes du couvert végétal élaborées à partir de la photo interprétation:

Afin d'évaluer les superficies disparues du domaine boisé, les deux cartes de végétation issues de la photo-interprétation ont fait l'objet d'une planimétrie à l'aide du logiciel QGis 2.10. Les deux planimétries ont donné les résultats suivants:

Planimétrie de la carte du couvert végétal de 1986:

Les résultats de la planimétrie de la carte d'occupation du sol obtenue à partir de la photo-interprétation des photographies aériennes de la mission de 1986 sont rapportés dans la figure 3. 


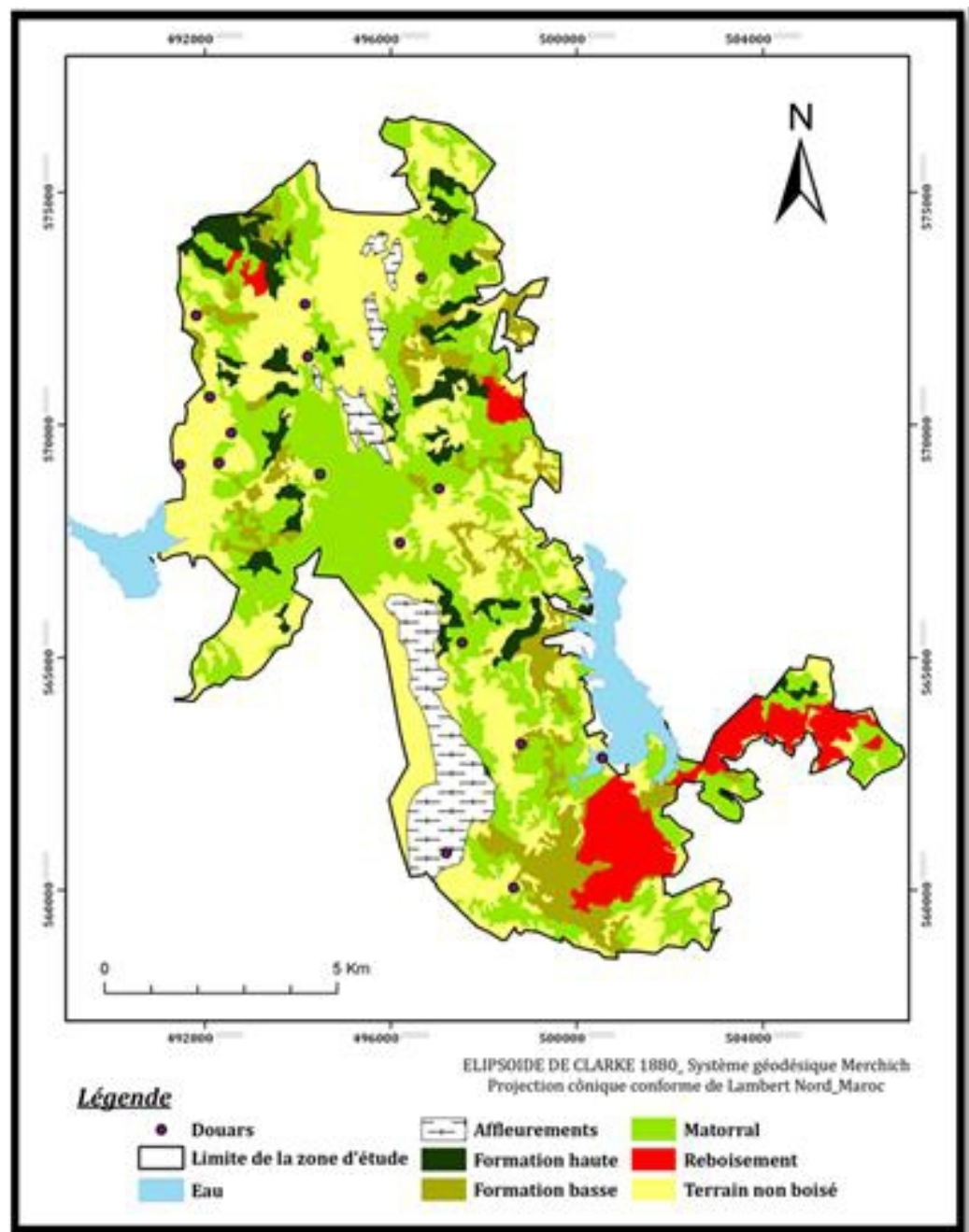

Figure 3 : Carte d'occupation du sol de la zone d'étude en 1986.

Planimétrie de la carte du couvert végétal de 2013:

La carte d'occupation des sols de l'année 2013 à été réalisée en se basant sur le traitement des images satelitaires précitées. Cette opération est conjuguée à des verifications sur le terrain dans le but de rectifier certaines unités présentant des confusions avec d'autres sur le plan physionomique. Les résultats de la planimétrie de cette carte sont présentés dans la figure 4. 


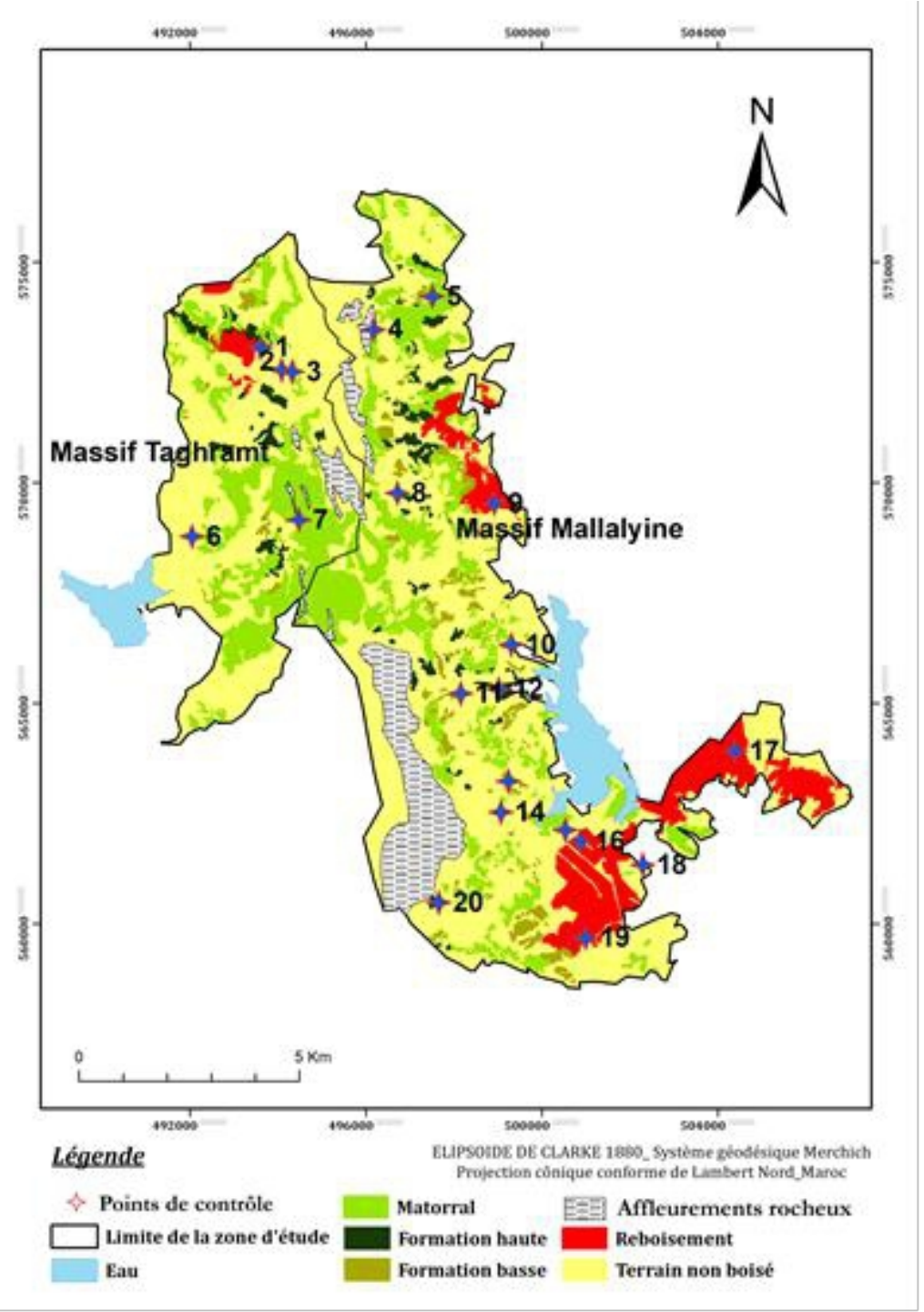

Figure 4 : Carte d'occupation du sol de la zone d'étude en 2013.

- Evaluation du changement intervenu dans le couvert végétal entre 1986 et 2013

L'effet des actions anthropiques sur les écosystèmes naturels se traduit par la dégradation de certaines formations au profit d'autres (Figure 5). On site comme exemple une agriculture de subsistance qui peut être dénudante au détriment d'un matorral. Pour bien visualiser ce genre de changement intervenu dans le couvert végétal des deux massifs forestiers de Mallalyine et Taghramt entre 1986 et 2013, on présentera dans les figures suivantes la répartition de l'ensemble des strates en 1986 et en 2013. 
Dans le but de mettre en évidence l'ampleur de cette dégradation, nous avons essayé d'évaluer la régression du couvert végétal durant ces vint-sept dernières années (1986-2013). L'étude comparative va être basée sur la superposition des deux cartes du couvert végétal (1986 et 2013). Ceci a été réalisé par traitement numérique grâce à un système d'information géographique. Si on compare l'état du couvert végétal entre 1986 et 2013 et si on élimine les matorrals et les terrains non boisés on remarque que le recul forestier est estimé à $60 \%$, donc c'est plus de la moitié du domaine forestier qui a disparu (Figure 6, Figure 7).

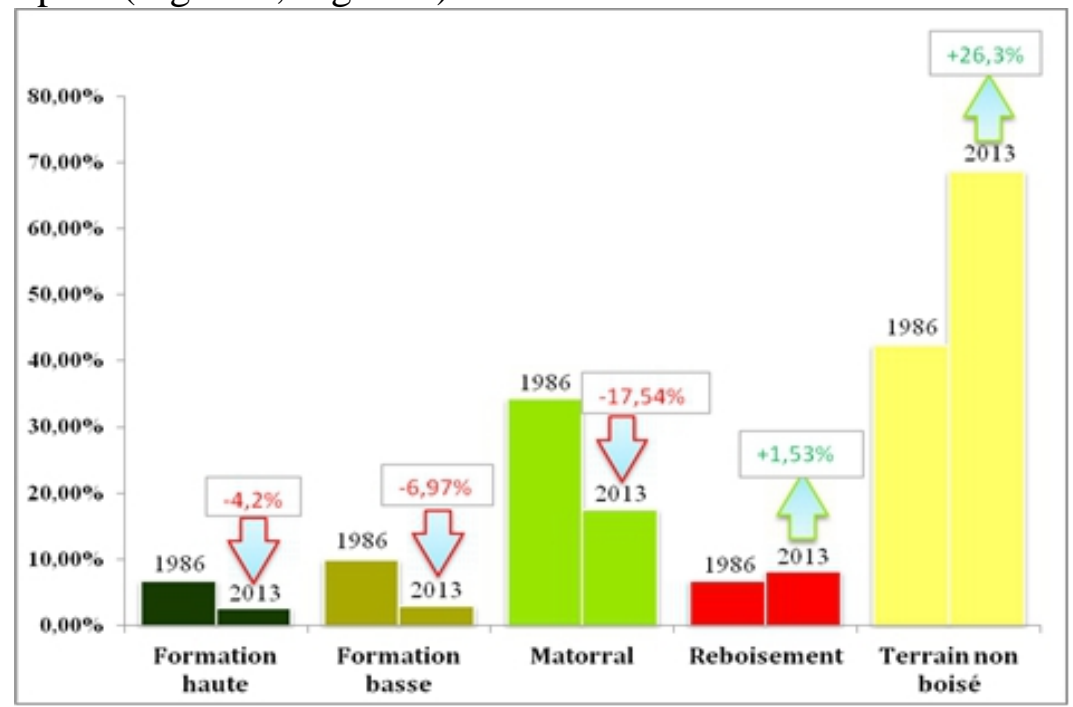

Figure 5 : Evaluation du changement intervenu dans le couvert végétal entre 1986 et 2013.

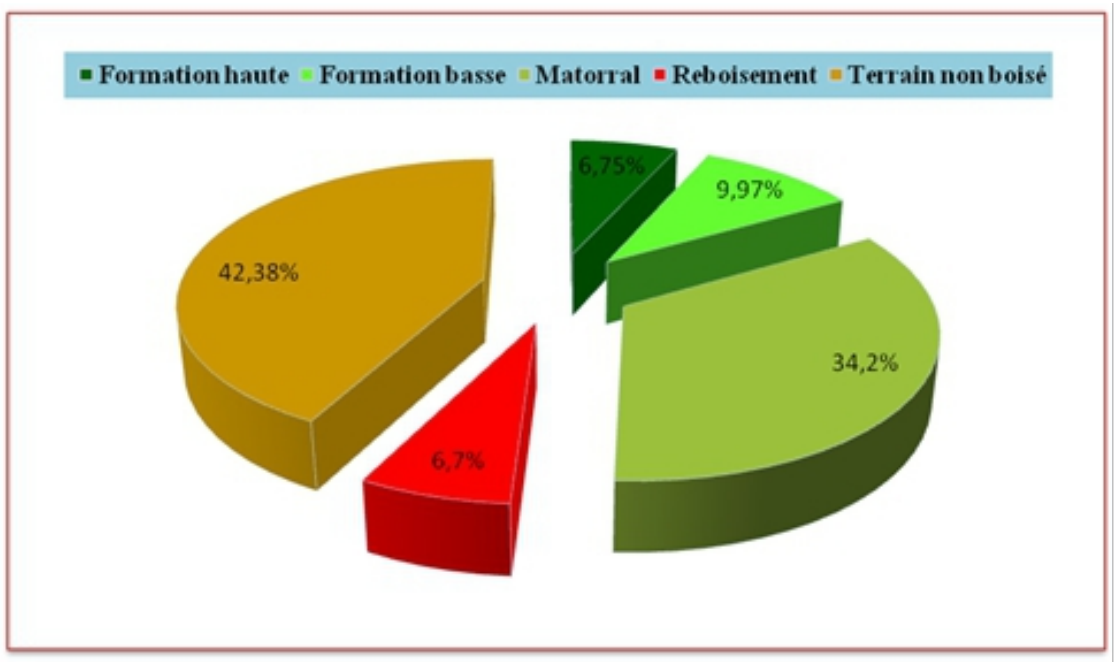

Figure 6 : Répartition des strates élaborées à partir des photographies aériennes de 1986. 


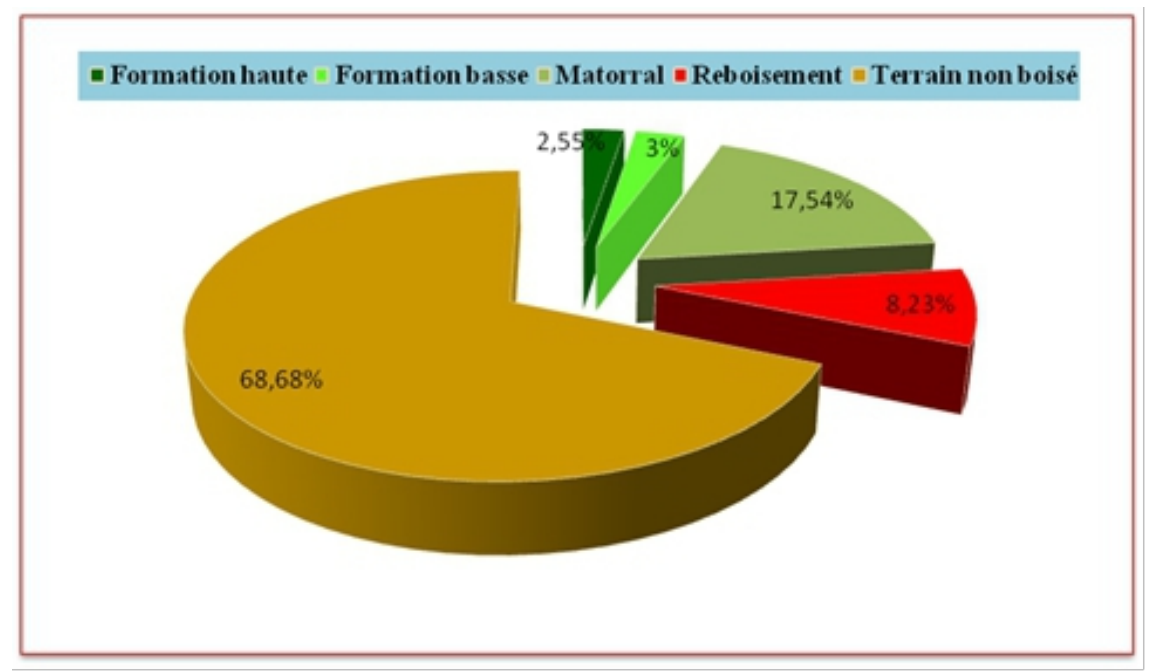

Figure 7: Répartition des strates élaborées à partir des images Google Earth 2013.

La superposition des deux cartes a mis en évidence les différentes transformations subies par chaque strate. Au terme de cette analyse nous avons dégagé les points essentiels suivants:

\section{Les formations hautes:}

La superficie qu'occupait cette strate en 1986 est de 770,8 ha alors qu'en 2013 elle ne couvre que 292 ha. En prenant comme référence la strate de 1986 soit donc une diminution de $62 \%$. La surface qui a disparu de cette strate et qui est de l'ordre de 478,8 ha soit $4,2 \%$ est passée vers d'autres strates présentant des stades de dégradation très avancée, à savoir:

- Matorral: 61 ha soit $8 \%$

- Reboisement: 38,5 soit $5 \%$

- Terrain non boisé: 379,3 soit $49 \%$

Ceci montre que presque la moitié de cette strate a été convertie au terrain non boisé.

\section{Les formations basses:}

Ces formations qui occupaient en 1986 une superficie de 1138 ha ne comptent en 2013 que 342 ha soit $3 \%$ de la superficie totale en 1986. La partie qui a disparu qui est de l'ordre de $6,97 \%$ se répartit comme suit:

- Matorral: 167 ha soit $15 \%$

- Reboisement: 9 ha soit $0,8 \%$

- Terrain non boisé: 250 ha soit $22 \%$

- Et une superficie de l'ordre de 28 ha soit 3\% évoluait pour devenir une formation haute. 


\section{Le matorral:}

Il dérive des types précédents par dégradation. Ce matorral a été répandu sur 3908 ha en 1986, mais actuellement il ne compte que 2004 ha soit la moitié $(51,3 \%)$ de la superficie initiale (1986).

Une superficie de l'ordre de 853 ha a été reboisé essentiellement par les pinèdes, l'Acacia cyanophyla et l'Eucalyptus camaldulensis. Le reste se répartit ainsi: 1000 ha pour le terrain non boisé et 51 ha évoluait en formation basse.

\section{Les reboisements:}

Les reboisements qui occupaient 765,7 ha en 1986 occupent actuellement 940,5 ha soit une faible progression de 1,53\%. Ils longent la partie Sud-est et la partie Nord de la zone d'étude. Ces reboisements visent la reconstitution des écosystèmes naturels à savoir le chêne liège et le thuya ainsi qu'à agrémenter le milieu par le reboisement des résineux surtout le pin pignon et le pin maritime.

\section{Terrains non boisés:}

Au cours de cette même période les terrains non boisés sont passés de 4842 ha à 7846 ha soit donc une augmentation de 26,3\%. Il faut toutefois distinguer dans cette strate les agglomérations, les affleurements rocheux et les terrains de culture. Les terrains de culture situés à proximité des habitations, souvent clôturés par des haies de cactus, complantés d'arbres fruitièrs. Ces terrains reçoivent une fumure plus importante, réservés à la production de légumes pour la consommation familiale. La culture céréalière est pratiquée dont le blé dur représente la culture d'hiver la plus importante. Ce gain de 3004 ha en superficie des terres de cultures pendant les vint-sept années réalisé au détriment du couvert végétal naturel (hautes formations, basses formations, matorral) est une menace sérieuse contre la protection des sols de l'érosion hydrique. La situation est d'autant plus grave que la majorité des terrains sont en pente. En effet la crête de la dorsale calcaire d'El Haouz se caractérise par des pentes très fortes supérieures à 30\% (Figure 8). 


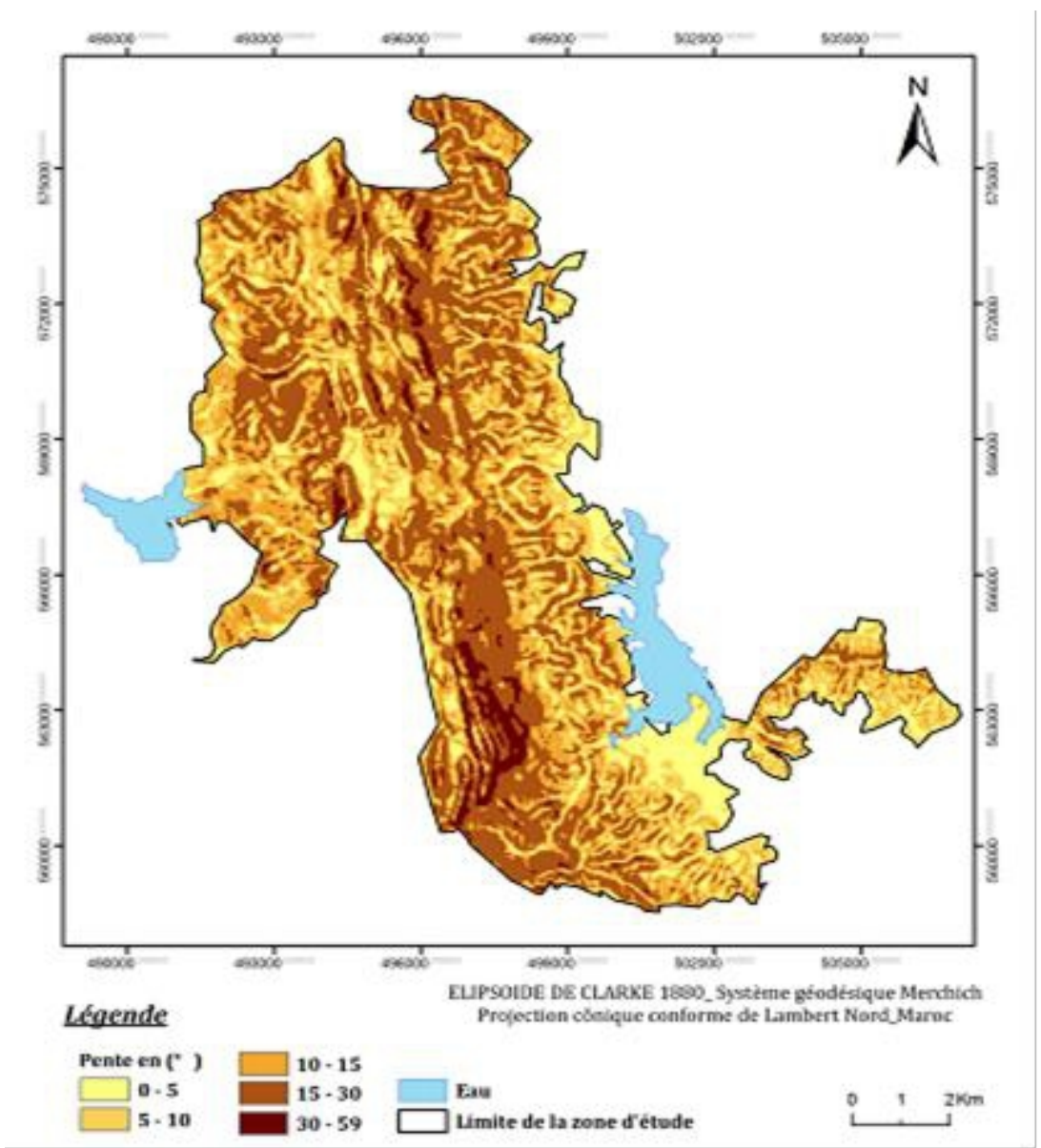

Figure 8: Carte des pentes de la zone d'étude.

\section{- Les facteurs de dégradation de la végétation}

La végétation des massifs forestiers de Mallalyine et Taghramt a été l'objet d'une dégradation dû essentiellement à:

Incendies en forêt :

Les incendies de forêt dans cette partie du rif occidental sont relativement plus importants que dans les autres régions du Maroc et ils causent des dommages considérables.

La moyenne d'incendies dans le massif forestier de Mallalyine et Taghramt est de l'ordre de 4 à 5 incendies par an. Le service provincial des eaux et forêts a estimé que la superficie dégradée atteint jusqu'à 50ha/an.

\section{Défrichements :}

Le défrichement gagne de l'ampleur surtout dans les régions isolées où la surveillance est défaillante. 
Ce facteur est responsable de la dégradation forestière de près de 20ha/an dans ces massifs. Le service provincial des eaux et forêts a enregistré 27 Procès Verbaux par année.

Dans la zone d'étude, il existe des espaces qui sont des terrains domaniaux ou en contentieux avec les collectivités ethniques ou encore des particuliers.

\section{Parcours :}

L'élevage constitue la première source de revenue agricole pour certains foyers de cette zone. Cependant, cette activité est entravée par la topographie accidentée, la charge caillouteuse des terrains, et des vents violents (Chergui) que connait la région. Ces facteurs freinent le développement de l'agriculture et empêchent une meilleure production des cultures. Des troupeaux constitués de Bovins (25,6\%), ovins (31\%) et caprins $(43,4 \%)$ parcourent la forêt durant toute l'année. Le pâturage constitue la principale cause de la dégradation des écosystèmes forestiers par prélèvement d'une biomasse importante par broutage puis piétinement qui empêchent toute régénération naturelle des essences sylvatiques. Le Service Provincial des Eaux et Forêt a enregistré une moyenne de 10 procès verbaux contre les propriétaires des troupeaux. La superficie de forêt dégradée est d'environ de 12ha en moyenne par année.

\section{Coupe du bois:}

L'une des causes principales de la dégradation des forêts a été la coupe régulière et systématique, entreprise avant l'indépendance par l'administration forestière espagnole (Benabid, 1982). Les récoltes de bois effectuées d'une manière anarchique parfois systématique dépassent de très loin celles autorisées au niveau des exploitations régulières. Ces dernières, se pratiquent irrationnellement et sans respect des règles sylvicoles, indispensable pour assurer la régénération naturelle des peuplements (Benabid, 1982). Le développement du groupement Olea europea sur les terres les plus faciles à cultiver explique largement les raisons de sa presque destruction totale. Les prélèvements délictueux du bois s'effectuaient dont le but de satisfaire des besoins le plus souvent de subsistance. Le haut commissariat des eaux et forêts et de la lutte contre la désertification a enregistré une moyenne de 35 procès verbaux et une superficie dégradée de 15ha par année. La coupe du bois prend le troisième rang après les incendies et les défrichements soit respectivement $52 \%$ et $21 \%$ et $15 \%$ de point de vue dégradation du couvert végétal. 


\section{Conclusion}

Comme indiqué dans l'introduction, le présent travail a pour but de mettre en exergue l'évolution du couvert végétal au niveau des massifs forestiers de Mallalyine et Taghramt (Rif occidental, Maroc). Deux cartes d'occupation du sol par photo-interprétation des photographies aériennes mission 1986 et des images Google Earth 2013, ont été élaborées.

Les deux cartes obtenues ont été traitées dans un système d'information géographique, ceci a permis d'évaluer les changements survenus au sein de chaque entité cartographiée. Ainsi, une régression du couvert végétal dans les vingt sept dernières années, a été mise en évidence. L'évaluation des deux cartes de 1986 et 2013 a montré que le domaine forestier connait un recul de $60 \%$; c'est donc plus de la moitié du domaine forestier de 1986 qui a disparu. En effet les formations hautes, les formations basses et le matorral ont régressé respectivement de $62 \%, 70 \%$ et $49 \%$ entre les années 1986 et 2013, tandis que les terrains non boisés et les reboisements se sont accrus respectivement de $26,3 \%$ et $1,53 \%$ par rapport à la superficie qu'ils occupaient en 1986. Les principaux facteurs contribuant à la régression du couvert végétal sont: la déforestation (35 délits de coupe par/an avec une superficie dégradée de l'ordre de 15 ha/an), le défrichement (27 délits par/an avec une superficie dégradée de 1'ordre de 20 ha/an), le surpâturage (10 délits de pâturage illicite par/an avec une superficie dégradée de l'ordre de 12 ha/an), les incendies de forêts (de 4 à 5 incendies par/an avec une superficie dégradée de l'ordre de 50 ha/an). En plus de ces facteurs, s'ajoute l'érosion hydrique. Il faut noter par ailleurs que le temps accordé à la réalisation de ce travail, n'a pas permis d'intégrer des études détaillées sur les composantes techniques, économiques et sociales. Ces composantes sont des facteurs déterminants pour expliquer la dégradation du couvert végétal dans ces deux massifs forestiers. Toutefois, les résultats obtenus constituent un outil de base auquel on peut se référer pour raisonner des actions à entreprendre en vue de minimiser la dégradation des ressources naturelles et améliorer la situation qui prévaut dans cette zone.

\section{Remerciéments}

Nous tenons à remercier et à témoigner toute notre reconnaissance aux personnels de la DREFLCD-SEAP du Rif, pour les commentaires et les discussions fructueuses qui ont permis l'amélioration de ce travail. Les évaluateurs anonymes sont remerciés pour leurs commentaires et suggestions.

\section{References:}

1. Administration des Eaux et Forêts et de la conservation des sols (AEFCS), (1995). Stratégie du développement forestier. 
Administration des eaux \& forets et de la conservation des sols, Rabat. $97 \mathrm{p}$.

2. Agence Nationale de la Conservation Foncière du Cadastre et de la Cartographie (ANCFCC)- Direction de la cartographie, (2007).

3. Benabid A., (2002). Le Rif et le Moyen Atlas (Maroc): Biodiversité, menaces, préservation. African Mountains High Summit Conference Nairobi, Kenya. 6-10 Mai 2002.

4. Benabid A., (2000). Flore et écosystèmes du Maroc: évaluation et préservation de la biodiversité. Ibis Press Paris. 359p.

5. Benabid A., (1984). Etude phytoécologique des peuplements forestiers et préforestiers du Rif Centro-occidental (Maroc). Trav. Inst. Sci. Rabat, Ser. Bot. 34. 64p.

6. Benabid A., (1982). Etude phyto-écologique biogéographique et dynamique des associations et séries sylvatique du Rif occidental. Problèmes posés par la reforestation et l'aménagement des peuplements forestiers actuels. Thèse. Univ. Droit, Econ., AixMarseille III. France. 165p.

7. Berkat O., (1995). Ressources naturelles: place de la recherche pastorale, projet Appui scientifique aux aménagements sylvopastoraux. In: Rejdali M., ed. Gestion des ressources pastorales. IAV Hassan II Rabat, Maroc. 165p.

8. Direction de la Conservation Foncière et des Travaux Topographiques (DCFTT) - Division de la carte, (1970).

9. El Gharbaoui A., (1986). L'homme et la montagne dans la dorsale calcaire du Rif (Maroc septentrional). In: Revue de l'Occident musulman et de la Méditerranée, N41-42, 1986. pp: 197-208.

10. El Gharbaoui A., 1981. La terre et l'homme dans la péninsule Tingitane; étude sur l'homme et le milieu naturel dans le Rif occidental. Trav. Inst. Scient., série géologie et géographie physique, 15, 439p.

11. El Haddad M., (1993). Etude et cartographie du couvert végétal du B.V. de Tleta : Utilisation des données SPOT HRV, des photographies aériennes et d'un S.I.G. Mémoire de 3ème cycle. Option: Eaux et Forêts. Institut Agronomique et Vétérinaire Hassan II. 120p.

12. FAO, (2013). Etat des forêts méditerranéennes 2013. Rome. E-ISBN 978-92-5-207538-7. www.fao.org/docrep/017/i3226f/i3226f.pdf.

13. Fennane M. \& Ibn Tattou M., (2005). Flore vasculaire du Maroc, inventaire et chorologie. Vol. 1, Pteridophyta, Gymnospermae, Angiospermae p.p. Trav. Inst. Sci. Rabat, Ser. Bot. 37. 483p.

14. Fennane M., (2003). Evolution actuelle et future des écosystèmes arborés au Sud de la Méditerranée : cas du Maroc. - Bocconea 16(1). pp: 439-444. - ISSN 1120-4060. 
15. Fennane M. \& Ibn Tattou M., (1998). Catalogue des plantes vasculaires rares, menacées ou endémiques du Maroc. - Bocconea 8. pp: 5-243.

16. Kouraimi B., (1997). Cartographie et analyse de la production pastorale et ligneuse du matorral dans le Rif Occidental: Cas du Bassin Versant de Tleta. Mémoire de 3ème cycle. Option: Pastoralisme. Institut Agronomique et Vétérinaire Hassan II. 109p.

17. Marien J.N., Billand A., (2009). Les systèmes socio-écologiques forestiers méditerranéens face aux changements globaux - Quelques questions posés par la filière bois énergie au Maroc, Forêt Méditerranéenne - tome XXX - n 4. pp: 297-300.

18. M'hirit O. \& Benchekroun F., (2006). Les écosystèmes forestiers et périforestiers : situation, enjeux et perspectives pour 2025. Rapport général, 50 ans de développement humain \& perspectives 2025. Contributions GT8. pp: 395-468.

19. Narjisse H., M'hirit O., Askarn O., Benzyane M. \& El Omerani A., (2001). Le couvert végétal marocain: etat de dégradation, acquis et perspectives en matière de conservation et de mise en valeur. Revue H.T.E. $\mathrm{N}^{\circ}$ 119. pp: 25-29.

20. Qarro M., (1996). Les ressources pastorales des massifs forestiers de Bab Berred et de Tanghaya-Kort. Rapport de projet "Protection et gestion participative des écosystèmes forestiers du Rif". ENFI, Salé. Maroc.

21. Tillier S., (2011). Gérer durablement la forêt méditerranéenne : exemple du parc naturel régional des Alpilles. Thèse de doctorat en Géographie. Université du Maine. pp: 389. 